\title{
Reanimación cardiopulmonar en época COVID-19, en el periodo postoperatorio
}

\author{
Cardiopulmonary resuscitation in COVID-19 \\ era, in the postoperative period
}

José Rodrigo Fernández Soto, ${ }^{*}$ Verónica Ruiz Vasconcelos, ${ }^{*}$ Aniza Surinam González Lumbreras, ${ }^{*}$
José Gamaliel Velazco González, ${ }^{\ddagger}$ Gerardo Esteban Álvarez Reséndiz ${ }^{\ddagger}$
Citar como: Fernández SJR, Ruiz VV, González LAS, Velazco GJG, Álvarez RGE. Reanimación
cardiopulmonar en época COVID-19, en el periodo postoperatorio. Acta Med Grupo cardiopulmonar en época COVID-19, en el periodo postoperatorio. Acta Med Grupo
Angeles. 2021; 19 (3): 387-391. https://dx.doi.org/10.35366/101733

\section{Resumen}

En la actualidad, todo paciente quien requiera intervención quirúrgica de urgencia debe ser considerado sospechoso de infección por SARS-CoV-2 y ser manejado de acuerdo con esta condición. El contagio por ese coronavirus tiene implicaciones potencialmente peligrosas en el periodo postoperatorio tanto para el enfermo como para el equipo humano que le da atención. Se estima que la mortalidad tras la operación, en enfermos con diagnóstico por SARS-CoV-2, es de $27.5 \%$. En contagiados por ese virus, la reanimación cardiopulmonar se considera un procedimiento de alto riesgo para el personal que da cuidados en el área de recuperación, dado lo cual es absolutamente necesario llevar a cabo el protocolo hospitalario de prevención. protocolo de prevención hospitalario, reanimación cardiopulmonar, choque séptico.

\section{INTRODUCCIÓN}

En la actualidad, cualquier paciente quien requiera intervención quirúrgica de emergencia debe ser considerado sospechoso de infección por SARS-CoV-2. ${ }^{1,2}$ En un estudio realizado en Wuhan, China, se encontró que, de 136 contaminados por tal coronavirus, la supevivencia a 30 días postparo cardiopulmonar fue de $2.9 \%$. $^{3}$ La reanimación
Palabras clave: COVID-19, equipo de protección personal,

\section{Abstract}

During the pandemic, every person in need of an emergent surgical procedure must be considered as possibly being infected by SARS-CoV-2 and receive care according to local preemptive protocols. SARS-CoV-2 ridden patients are at increased risk of adverse outcomes during the postoperative period and represent a hazard for healthcare personnel. Postoperative mortality among those contaminated with this coronavirus is estimated to be around $27.5 \%$. Cardiopulmonary reanimation in patients infected by this virus is considered a high risk procedure for the attending personnel in the recovery room and it is of paramount importance to establish and implement a local preventive protocol.

Keywords: COVID-19, personal protection equipment, local preventive protocol, cardiopulmonary resuscitation, septic shock.

cardiopulmonar (RCP) en infectados con SARS-CoV-2 se considera un procedimiento de alto riesgo, dado lo cual es indispensable llevar cabo los protocolos de prevención de los trabajadores en la atención de esos dolientes. ${ }^{4}$

En este trabajo se mencionará el método establecido para pacientes bajo sospecha de contagio por SARS-CoV-2 en el Hospital Ángeles Lomas, en un varón internado sufriendo enfermedad diverticular Hinchey III. ${ }^{5}$

Correspondencia:

José Rodrigo Fernández Soto

Correo electrónico: rodrigoferso21@gmail.com

Hospital Ángeles Lomas. México.
Aceptado: 25-01-2021.

www.medigraphic.com/actamedica

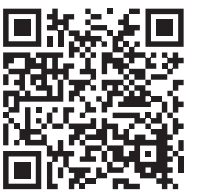




\section{PRESENTACIÓN DE CASO CLÍNICO}

Masculino de 65 años, con antecedente de adenocarcinoma de próstata, hipertensión arterial sistémica y dislipidemia, en tratamiento médico. Inicia padecimiento actual 72 horas previas a su ingreso a urgencias, con aumento no cuantificado de temperatura, dolor abdominal generalizado, de comienzo súbito, intensidad 10/10, acompañado de náusea y vómito. En su domicilio recibió atención médica sin presentar mejoría, sucedido ésto, acude a emergencias.

A su llegada al servicio, evidenció tensión arterial de $60 / 44 \mathrm{mmHg}$, frecuencia cardiaca (FC) $110 \mathrm{lpm}, \mathrm{SpO}_{2}$ de $85 \%$ al aire ambiente, temperatura $38.5^{\circ} \mathrm{C}$, frecuencia respiratoria (FR) $26 \mathrm{rpm}$. A la exploración física dirigida, reveló signos de deshidratación y palidez generalizada, campos pulmonares bien ventilados, ruidos cardiacos rítmicos sin fenómenos agregados, peristalsis ausente, timpanismo generalizado, dolor a la palpación superficial y profunda de predominio en hemiabdomen izquierdo con resistencia muscular involuntaria, acompañados de signo de rebote positivo. Se inicia tratamiento médico y reanimación hídrica, sin lograr aumento de la presión arterial, por lo que se inició apoyo usando norepinefrina. Laboratorios arrojaron leucocitosis, bandemia y creatinina de $1.52 \mathrm{mg} / \mathrm{dL}$.

Se tomó gasometría venosa, como se muestra en la Tabla 1. En el análisis tomográfico toracoabdominal se reportó con diverticulitis Hinchey III, sin datos pulmonares sugestivos de infección por SARS-CoV-2 (Figuras 1 y 2).
Se programa de urgencia una laparoscopia exploradora, bajo el protocolo de paciente con sospecha de inoculación por SARS-CoV-2, que consiste en: quirófano exclusivo, material de protección al personal hospitalario, recomendado en la guía para el manejo perioperatorio de enfermos con contagio por dicho coronavirus. ${ }^{6}$

En quirófano se realizó inducción de secuencia rápida empleando propofol, fentanilo, rocuronio y lidocaína, intubación endotraqueal usando videolaringoscopio, conectándose a ventilación controlada por volumen. Se colocó catéter venoso central yugular izquierdo y línea arterial radial. Se obtuvo gasometría arterial en el transcurso de la operación con los parámetros plasmados en la Tabla 1. Siguió el mantenimiento de la anestesia, aplicando infusión de fentanilo, lidocaína y desfluorano. Reposición de líquidos de acuerdo a monitoreo de presión venosa central; se suspendió y retiró paulatinamente la medicación con noradrenalina.

Inicialmente, la intervención fue laparoscópica; sin embargo, debido a la poca visibilidad de estructuras anatómicas, se convirtió a cirugía abierta. Terminó procedimiento quirúrgico, el cual tuvo una duración de 3 horas 45 minutos. Se decidió extubar al doliente y se recuperó en sala de quirófano con escala Alderete 9/10, hemodinámicamente estable, sin apoyo de vasopresor. Se deja suero analgésico conteniendo lidocaína 2,800 mg + ketamina $400 \mathrm{mg}$ para 24 horas, se administró dosis única de morfina $5 \mathrm{mg}$ intravenosa.

\begin{tabular}{|c|c|c|c|c|c|}
\hline & Urgencias & Quirófano & Postparo & UTI & UTI \\
\hline $\mathrm{FiO}_{2}(\%)$ & 21 & 40 & 60 & 70 & 70 \\
\hline Temperatura $\left({ }^{\circ} \mathrm{C}\right)$ & 36.7 & 36.5 & 35.9 & 36.9 & 36.9 \\
\hline $\mathrm{PaFi}\left(\mathrm{PaO}_{2} / \mathrm{FiO}_{2}\right)$ & & 238 & 119 & 118 & \\
\hline Tipo & Venosa & Arterial & Arterial & Arterial & Venosa \\
\hline $\mathrm{pH}$ & 7.41 & 7.34 & 7.06 & 7.34 & 7.30 \\
\hline $\mathrm{PaCO}_{2}$ (Torr) & 30.8 & 38.2 & 57.6 & 30.6 & 38.1 \\
\hline $\mathrm{SO}_{2}(\%)$ & 77.8 & 97.0 & 84.2 & 95.3 & 65.5 \\
\hline $\mathrm{PaO}_{2}(\mathrm{mmHg})$ & 42.4 & 95.0 & 71.1 & 82.8 & 37.4 \\
\hline $\mathrm{Hb}(\mathrm{g} / \mathrm{dL})$ & 11.5 & 11.4 & 12.6 & 17.9 & 18.1 \\
\hline Glucosa (mg/dL) & 143 & 163 & 277 & 292 & 219 \\
\hline Lactato (mmol/L) & 3.5 & 1.9 & 8.9 & 4.6 & 4.8 \\
\hline $\mathrm{HCO}_{3}$ & 20.8 & 20.9 & 13.1 & 17.9 & 18.1 \\
\hline p50 (mmHg) & 26.7 & 29.0 & 40.4 & 28.5 & 27.8 \\
\hline cBase (Ecf)c & -4.4 & -4.2 & -12.7 & -8.0 & -7.4 \\
\hline Anión GAP & 11.5 & & 10.6 & 10.0 & 10.9 \\
\hline
\end{tabular}


Después de una hora, en forma súbita el enfermo presentó deterioro del estado de alerta y depresión respiratoria sin respuesta a estímulos; se corroboró presencia de latido carotideo. Se registraron los siguientes signos vitales: $\mathrm{SpO}_{2}$ de $60 \%$ con puntas nasales a $4 \mathrm{~L} /$ min, tensión arterial 85/58 mmHg, FC de 55 lpm y ritmo sinusal. Se le asistió mediante oxigenación no invasiva utilizando mascarilla facial, sin mejoría de la oximetría de pulso. Se buscó nuevamente latido en carótide, al declararse ausente, se iniciaron maniobras de reanimación avanzadas en pacientes COVID (+) o sospechoso, según el soporte vital cardiovascular avanzado (ACLS), con actividad eléctrica sin pulso. Se colocó tubo endotraqueal con videolaringoscopio, logrando ritmo sinusal después de cuatro ciclos de reanimación cardiopulmonar (RCP). Se iniciaron cuidados postparo usando soluciones cristaloides en carga, persistiendo con hipotensión, dado lo cual se administró infusión de norepinefrina a $0.6 \mu \mathrm{g} /$ $\mathrm{kg} / \mathrm{min}$, sulfato de magnesio un gramo, gluconato de calcio al $10 \%$ una ámpula, introducción de fentanilo $1.6 \mathrm{\mu g} / \mathrm{kg} / \mathrm{min}$. Se tomó gasometría arterial (Tabla 1) y radiografía de tórax (Figura 3).

El afectado fue trasladado a la Unidad de Cuidados Intensivos, donde al recibirlo se adquirió gasometría arterial y venosa, arrojando los valores expuestos en la Tabla 1.

A lo largo de su estancia en la Unidad de Terapia Intensiva, mediante prueba PCR, el enfermo fue dictaminado con contagio por SARS-CoV-2. Permaneció recibiendo medidas de neuroprotección y ventilación mecánica invasiva de auxilio pulmonar durante cinco días, alcanzando evolución satisfactoria, por lo que el paciente egresó de la terapia intensiva neurológicamente sin secuelas. Fue dado de alta del hospital 17 días posterior al evento.

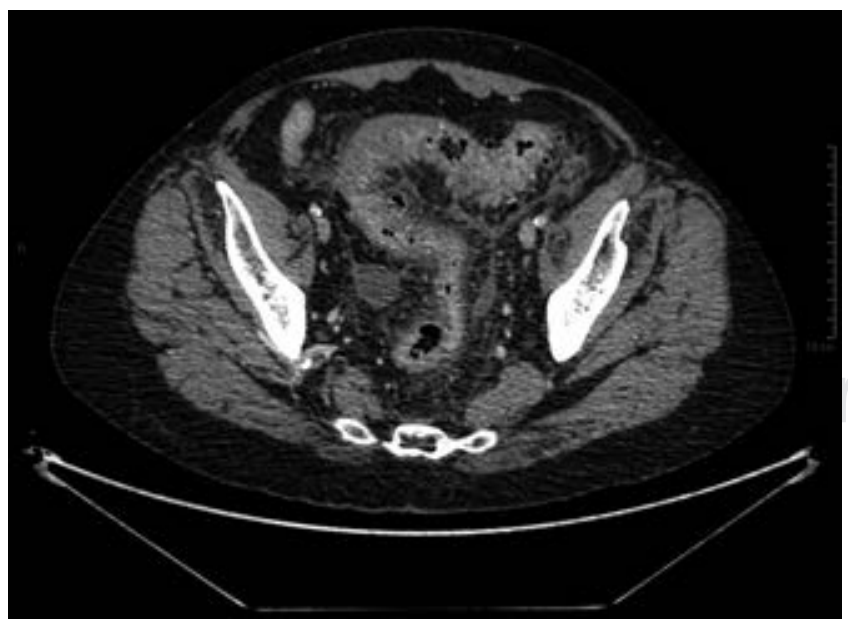

Figura 1: Tomografía computarizada, corte axial, con diverticulitis, aire libre en cavidad abdominal, Hinchey III.

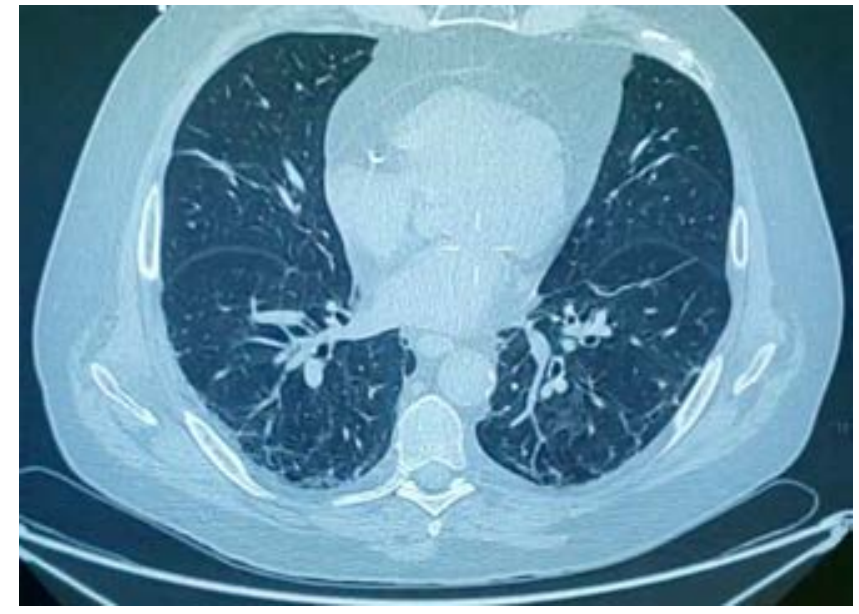

Figura 2: Tomografía computarizada, corte axial.

\section{DISCUSIÓN}

Los afectados diagnosticados con SARS-CoV-2 se han asociado a un mal pronóstico en el periodo postoperatorio, esto se debe a diversos factores, por ejemplo la anestesia general, ventilación mecánica, transfusión o pérdida sanguínea y sobre todo a raíz de la respuesta inflamatoria sistémica asociada a la maniobra quirúrgica. Además, puede ser complicado realizar el diagnóstico de infección de COVID-19 en el lapso posterior a la operación, debido a que los síntomas se pueden atribuir a otras condiciones más comunes tras una intervención. ${ }^{8}$ En una investigación, realizada por Moliere y colaboradores, en donde se evaluaron a 46 infectados por ese virus con síntomas agudos en ese intervalo, se verificó que 25 \% falleció a consecuencia de dificultades tanto postoperatorias como a causa de tropiezos generados por la propia infección. ${ }^{9}$

Como se expuso en el caso clínico relatado, el paciente acudió presentando una situación clínica que ameritaba manejo quirúrgico de urgencia. Al ser parte del protocolo, se obtuvo la prueba de PCR, mediante hisopado nasal y faríngeo, sin embargo, la emergencia del cuadro clínico no permitió esperar el resultado de dicho examen. El grupo médico realizó la cirugía con el equipo de protección personal (EPP) completo, asimismo, el lapso tras la intervención se sucedió en sala de quirófano, como se menciona en las recomendaciones internacionales. $., 10,11$

Actualmente, existe muy poca información sobre la tasa de mortandad y advenimiento de complicaciones en enfermos con SARS-CoV-2 quienes se someten a intervención. En una investigación efectuada por Doglietto y coautores, se estudió como objetivo primario la mortalidad quirúrgica temprana y las contratiempos postoperatorios en infectados con tal virus. ${ }^{12}$ En dicho estudio se analizaron 41 pacientes, a los cuales se les 
realizó cirugía principalmente de urgencia. De este total de afectados, 33 (80.5\%) tenían un análisis preoperatorio positivo para SARS-CoV-2, mientras que ocho de ellos (19.5\%) presentaron prueba positiva en los cinco días posteriores a la operación.

Con respecto a las dificultades postoperatorias en pacientes con dicha infección, se encontró que las complicaciones pulmonares se manifestaron más frecuentemente en esa fase; asimismo, los percances trombóticos también tuvieron una alta incidencia. ${ }^{12}$

En un afectado con diagnóstico de SARS-CoV-2, se ha encontrado que las principales causas de paro cardiorrespiratorio son choque distributivo o cardiogénico, hipoxia y prolongación de intervalo QT, secundario a fármacos como la hidroxicloroquina o azitromicina. ${ }^{13}$ Con respecto al caso clínico, es probable que la causa de la suspensión cardiorrespiratoria haya sido hipoxemia, la cual pudo ser secundaria a la infección por el coronavirus asociado al proceso séptico y la ventilación mecánica invasiva.

En contagiados por SARS-CoV-2, la reanimación cardiopulmonar se considera un procedimiento que genera múltiples aerosoles, principalmente al momento de las compresiones torácicas, la oxigenación con presión positiva y la manipulación de la vía aérea, por lo cual la plantilla sanitaria que realiza RCP acusa mayor riesgo de infectarse. ${ }^{4,14}$ El objetivo de realizar RCP en paciente bajo sospecha o infectado de SARS-CoV-2, es regresarlo a circulación espontánea sin comprometer la seguridad de los trabajadores de salud. ${ }^{15}$

En un evento de paro cardiorrespiratorio presenciado, se recomienda que toda la cuadrilla sanitaria tenga el EPP adecuado antes de entrar a ejecutar RCP, a pesar de que esto pueda retrasar la atención del colapso, y se deberá

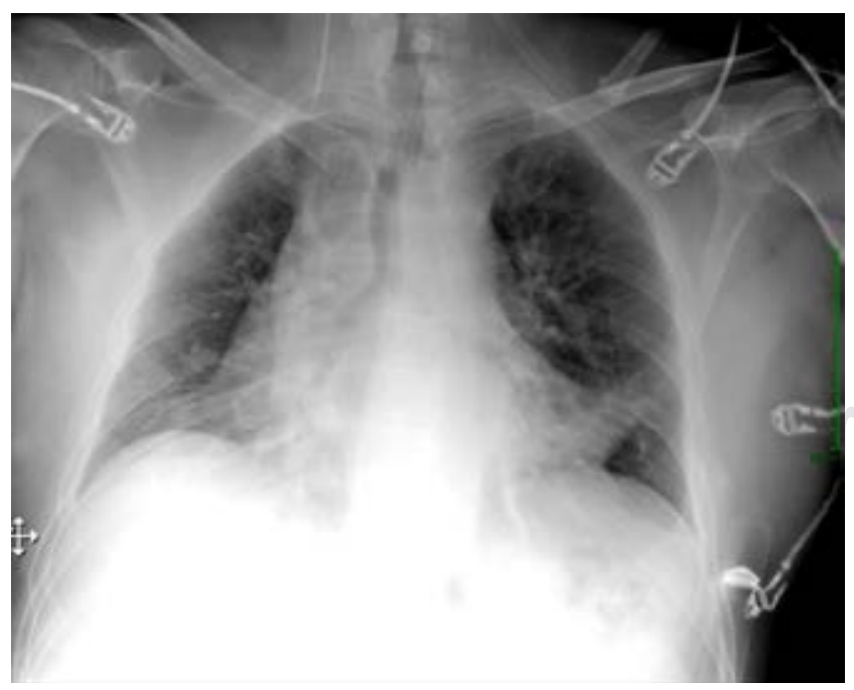

Figura 3: Radiografía de tórax anteroposterior. llevar a cabo la vigilancia del suceso empleando el menor número posible de trabajadores hospitalarios. ${ }^{15}$

Como se mencionó en el caso clínico, el paro cardiopulmonar fue presenciado y se iniciaron maniobras de forma inmediata, lo cual ayudó a que el pronóstico y evolución del enfermo fueran favorables. Uno de los aspectos más importantes sobre el caso clínico, es que la adaptación y protocolos implementados dentro de este hospital permitieron brindar una adecuada atención del paro cardiopulmonar con una evolución benigna para el paciente y para el personal de salud. ${ }^{16}$

\section{CONCLUSIÓN}

En estas épocas, en el contexto de la pandemia, antes de iniciar RCP en un paciente sospechoso o diagnosticado, es importante aquilatar algunos aspectos relevantes, por ejemplo la instauración del protocolo de atención a enfermos SARS-CoV-2, al igual que siempre asegurar la salvaguarda del equipo sanitario. En lo tocante al caso clínico presentado anteriormente, es posible concluir que al ser un evento presenciado y al contar con todos los procedimientos de protección adecuados, el cuidado del paro fue exitoso, considerando que era un enfermo potencialmente recuperable y quien actualmente se encuentra sin secuelas neurológicas postparo. Asimismo, el grupo humano de salud que estuvo presente durante el incidente no se contagió ni presentó síntomas posteriores. Por ese motivo, se puede considerar que tanto los protocolos de actuación que se modificaron por la actual pandemia, como la RCP, favorecieron un desenlace propicio para el enfermo.

\section{REFERENCIAS}

1. Coccolini F, Perrone G, Chiarugi M, Di Marzo F, Ansaloni L, Scandroglio I et al. Surgery in COVID-19 patients: operational directives. World J Emerg Surg. 2020; 15 (1): 25.

2. Nahshon C, Bitterman A, Haddad R, Hazzan D, Lavie O. Hazardous postoperative outcomes of unexpected COVID-19 infected patients: a call for global consideration of sampling all asymptomatic patients before surgical treatment. World J Surg. 2020; 44 (8): 2477-2481.

3. Shao F, Xu S, Ma X, Xu Z, Lyu J, Ng M et al. In-hospital cardiac arrest outcomes among patients with COVID-19 pneumonia in Wuhan, China. Resuscitation. 2020; 151: 18-23.

. DeFilippis EM, Ranard LS, Berg DD. Cardiopulmonary resuscitation during the COVID-19 pandemic: a view from trainees on the front line. Circulation. 2020; 141 (23): 1833-1835.

5. Tochigi T, Kosugi C, Shuto K, Mori M, Hirano A, Koda K. Management of complicated diverticulitis of the colon. Ann Gastroenterol Surg. 2017; 2 (1): 22-27.

6. Chen X, Liu Y, Gong Y, Guo X, Zuo M, Li J et al. Perioperative management of patients infected with the novel coronavirus: recommendation from the joint task force of the Chinese Society of Anesthesiology and the Chinese Association of Anesthesiologists. Anesthesiology. 2020; 132 (6): 1307-1316. 
7. AHA. ACLS cardiac arrest algorithm for suspected or confirmed COVID-19 patients. AHA. 2020.

8. Aminian A, Safari S, Razeghian-Jahromi A, Ghorbani M, Delaney CP. COVID-19 outbreak and surgical practice: unexpected fatality in perioperative period. Ann Surg. 2020; 272 (1): e27-e29.

9. Moliere S, Veillon F. COVID-19 in post-operative patients: imaging findings. Surg Infect (Larchmt). 2020; 21 (5): 416-421.

10. Dexter F, Parra MC, Brown JR, Loftus RW. Perioperative COVID-19 defense: an evidence-based approach for optimization of infection control and operating room management. Anesth Analg. 2020; 131 (1): $37-42$

11. Bresadola V, Biddau C, Puggioni A, Tel A, Robiony M, Hodgkinson $J$ et al. General surgery and COVID-19: review of practical recommendations in the first pandemic phase. Surg Today. 2020; 50 (10): 1159-1167.
12. Doglietto F, Vezzoli M, Gheza F, Lussardi GL, Domenicucci M, Vecchiarelli $L$ et al. Factors associated with surgical mortality and complications among patients with and without coronavirus disease 2019 (COVID-19) in Italy. JAMA Surg. 2020; 155 (8): 691-702.

13. Argüello-López B, Navarro-Vargas JR. Reanimación cardiopulmonar en pacientes con coronavirus SARS-CoV-2 en posiciones supino y prono. Rev Chil Anest. 2020; 49 (5): 605-613.

14. Meng L, Qiu H, Wan L, Ai Y, Xue Z, Guo Q et al. Intubation and Ventilation amid the COVID-19 outbreak: Wuhan's experience. Anesthesiology. 2020; 132 (6): 1317-1332.

15. Chahar P, Marciniak D. Cardiopulmonary resuscitation in COVID-19 patients. Cleve Clin J Med. 2020. doi: 10.3949/ccjm.87a.ccc040.

16. Prakash L, Dhar SA, Mushtaq M. COVID-19 in the operating room: a review of evolving safety protocols. Patient Saf Surg. 2020; 14: 30. 